\title{
EnCUestas de OpINIÓn PÚblica en AmÉrica LATINA: DESAFÍOS Y CONTROVERSIAS
}

\author{
María Braun ${ }^{1}$ (Argentina)
}

\begin{abstract}
A public opinion survey is, given the field in which it is involved -political communication- a controversial activity. It is also, particularly in countries that have experienced decades of institutional disruption, a relatively new, strongly questioned activity, where academia has played quite a marginal role. This paper reflects on the possible forms or paths that can contribute to professionalizing the field, to reinforcing its weak institutionalization and legitimizing surveys and the professional community that conducts them. To that end, we used data from a survey carried out by WAPOR Latinoamérica among Latin American professionals and academics linked to the study of public opinion.
\end{abstract}

Síntesis: Las encuestas de opinión pública son, por el campo en que se insertan -el de la comunicación política- una actividad controversial. Son también, particularmente en países que han sufrido décadas de rupturas institucionales, una actividad relativamente nueva, fuertemente cuestionada, donde el papel de la academia ha sido bastante marginal. El objetivo de este trabajo es reflexionar sobre formas o caminos posibles que contribuyan a profesionalizar

Socióloga, Universidad de Buenos Aires (UBA) y Máster en Desarrollo Urbano, University College London (UCL). Es socia fundadora y presidente de MBC MORI, empresa de investigación de mercado y opinión presente en la Argentina desde 1995, y preside la comisión directiva de la sede Buenos Aires de WAPOR Latinoamérica. Fue docente de la Universidad de Buenos Aires y de la Facultad Latinoamericana de Ciencias Sociales (FLACSO). Compiladora del libro Opinión pública, una mirada desde América Latina; tiene artículos publicados en el Centro Editor de América Latina, y en Desarrollo Económico, Crítica y Utopía, La Ciudad Futura, así como en otros diarios y revistas nacionales. Email:mariabraun@mbc-mori.com.ar. 
la disciplina, a reforzar su débil institucionalización, y a legitimar las encuestas y la comunidad profesional que las lleva a cabo. Para ello se utilizan los datos de una encuesta realizada por WAPOR Latinoamérica entre profesionales y académicos latinoamericanos ligados al estudio de la opinión pública.

\section{Introducción}

En los procesos electorales, cuando la comunicación política se intensifica, las encuestas de opinión pública y los especialistas que las llevan a cabo adquieren un particular protagonismo. Este protagonismo de las encuestas se combina muy frecuentemente con fuertes cuestionamientos: políticos que afirman no creer en ellas, sospechas de manipulación de los datos y hacia los encuestadores por parte de los partidos políticos o los medios de comunicación, desconfianza respecto de la validez de los pronósticos. Se cuestiona su creciente papel en los procesos electorales, se les atribuye capacidad para influir sobre el votante, y, en definitiva, se les reprochan o festejan sus errores o aciertos a la hora de los resultados.

Por otro lado, y más allá de estos cuestionamientos -algunos de los cuales se discutirán a lo largo de estas páginas-, no cabe duda de que las encuestas, como área de las ciencias sociales, han mostrado ser una herramienta versátil y potente para conocer las actitudes, opiniones y prácticas de segmentos importantes de la población, para describir valores, medir cambios y comparar sociedades. Tanto es así que, hasta cierto punto, puede decirse que el análisis de la opinión pública tiende a sustituir el análisis de la sociedad. En cuanto a las encuestas preelectorales -seguramente la rama más visible de la actividad-forman parte hoy del arsenal obligado de herramientas que se despliega en toda elección. 
El objetivo de este trabajo es reflexionar sobre algunas de las particularidades de esta área de conocimiento, relativamente nueva, de las ciencias sociales. Para ello me detendré, por un lado, en el señalamiento de ciertas lógicas de producción inherentes a la actividad y que la instalan, casi de un modo inevitable, en un campo de ambigüedades y contradicciones. Por el otro, y a partir de los datos de una encuesta entre profesionales de la opinión pública, describiré el lugar que los propios encuestadores le otorgan a su actividad, la imagen que tienen de ella y de la forma en que se ha ido conformando en estos países. La idea es que estas reflexiones y estas miradas sean un aporte para pensar acciones o líneas que puedan contribuir a profesionalizar la disciplina, a reforzar su débil institucionalización y a legitimar las encuestas de opinión pública y la comunidad profesional que las lleva a cabo.

\section{Una actividad que se instala en un campo de contradicciones y ambigüedades ${ }^{2}$}

Hoy es casi un lugar común hablar de opinión pública. Veinticinco años de democracia y una nueva y particular articulación entre medios de comunicación, sistema político y opinión pública crearon el escenario de visibilidad de esta última a través de su instrumento más popular: las encuestas preelectorales. Éstas se ajustaron a los requerimientos tanto de la dirigencia política, generando un conocimiento rápido de las demandas prevalecientes -fácilmente transformadas en slogans de campaña-, como de los medios de comunicación, generando una información atractiva y de fácil comprensión, muchas veces asimilable al hechizo de la posibilidad de conocer los acontecimientos antes de que se produzcan.

Una versión preliminar de estas cuestiones puede verse en Braun y Rovner (1999) y en Braun, Catterberg y Rovner (2009). 
Ahora bien, estas cualidades no deberían hacernos perder de vista que, en tanto técnica de obtención de conocimiento, las encuestas nacieron en el campo de las ciencias sociales. De ese modo, no les son necesariamente propias las lógicas de la estrategia electoral, ni las de la producción de acontecimientos. No les son propias, pero tampoco pueden sustraerse a ellas, precisamente porque comparten un espacio: el de la comunicación política, un espacio en el que -como dice Wolton (1995)- participan e intercambian discursos tres actores diferentes: los políticos, los periodistas y la opinión pública (que se expresa, sobre todo, a través de los sondeos). ${ }^{3}$ En ese espacio, las encuestas -pese a ocupar un papel relevante particularmente en épocas de elecciones- no se mueven con independencia de los políticos que las encargan ni de los medios que las difunden.

La comunicación política tiene, según Wolton, una mala imagen que deviene de la idea de que sería fácil operar sobre la opinión pública, manipularla. Existe efectivamente una creencia, bastante generalizada por cierto, de que el conocimiento de los resultados de las encuestas impacta sobre el comportamiento del votante, creencia que ha llevado en muchos países a establecer restricciones para su publicación. El estudio que desde 1984 realizan conjuntamente ESOMAR y WAPOR muestra que en 2003 existe en treinta países, casi la mitad de la muestra estudiada, algún tipo de restricción para la publicación de las

\footnotetext{
Según Wolton, la comunicación política debe ser pensada como la confrontación de los tres discursos característicos de la política: la ideología y la acción para los políticos; la información para los periodistas; y la comunicación para la opinión pública y los sondeos. Tres discursos que están en permanente tensión, ya que cada uno guarda parte de la legitimidad política democrática. Para los políticos, la legitimidad resulta de la elección; para los periodistas, está vinculada con la información; y respecto de los sondeos, "representantes" de la opinión pública, la legitimidad es de orden científico y técnico (Wolton, 1995).
} 
encuestas (ESOMAR / WAPOR, 2003). Un análisis del caso argentino (Alonso et al.), hecho en base a 81 iniciativas presentadas en el parlamento entre 1987 y 2006, muestra que en la mayoría de los casos la justificación de un marco normativo a la investigación por encuestas y la difusión de sus resultados está relacionada con la idea de una opinión pública manipulable y la pretensión de resguardar la democracia garantizando la transparencia del proceso electoral.

Que las encuestas tienen un papel relevante en la vida política de las democracias modernas, no cabe duda. Informaciones sobre la imagen de los dirigentes políticos o de la gestión del gobierno son de consumo casi obligado en las democracias modernas, y los estudios que toman en cuenta las opiniones, expectativas y necesidades de la población son cada vez más utilizados para el diseño de las políticas públicas. Que el papel de las encuestas se hace más visible en épocas eleccionarias también es evidente. Hoy es impensable una campaña política sin ellas: se las usa para auscultar climas, para diseñar campañas, para crear mensajes, para definir énfasis. También sabemos que se toman decisiones respecto de cuestiones tan relevantes como la definición de candidaturas con encuestas en la mano, y es más o menos obvio que la capacidad de recolección de fondos depende fuertemente de la posición del candidato en las encuestas. Por último, cualquiera que alguna vez haya estado relativamente involucrado en una campaña política, sabe que las expectativas, positivas o negativas, impactan con fuerza sobre el ánimo de los candidatos (recurso fundamental en cualquier campaña política). En síntesis: se toman decisiones, se definen estrategias, se modifica o intenta modificar la realidad haciendo un uso estratégico o táctico de la información que generan las encuestas.

No existen, sin embargo, resultados conclusivos acerca del impacto de las encuestas sobre el comportamiento del 
votante. Varios autores, usando diferentes tipos de metodologías, han trabajado sobre hipótesis de distintos tipos de efectos: desde el efecto bandwagon o de Panurgo (se vota al que se supone va a ganar y de esta forma se consolida la mayoría), hasta el underdog o de David (se vota al partido más débil, lo que beneficia al perdedor), o derrotista (no se vota). En cualquier caso, si este efecto existe, es débil, y tampoco es clara cuál es la dirección que asume. Además, seguramente varía según el tipo de votante: si se supone un votante racional e instruido puede pensarse que la encuesta lo provee de "asistencia interpretativa" -de información que le ayuda a interpretar la realidad- y que esta información puede tener tanto o más impacto que el discurso de los candidatos; si se supone en cambio un votante desinteresado de la política y/o poco instruido, es posible que impacte más sobre su comportamiento una noticia basada en testimonios, por ejemplo, que en cuadros y estadísticas (Donsbach, 2001). ${ }^{4}$

La relación entre medios de comunicación y comportamiento electoral es compleja y ha sido y es objeto de análisis teórico-político. En este sentido, siempre es útil recordar a Lazarsfeld y su teoría de los alcances limitados: para este sociólogo austríaco radicado en Estados Unidos, que fue además uno de los fundadores de la sociología empírica, los medios simplemente confirman o consolidan los procesos de formación de opiniones y actitudes que los individuos conforman a partir de sus entornos sociales. Y para este autor, el espacio determinante en la formación de las opiniones es el "grupo primario" o grupo de liderazgo social (Lazarsfeld y Katz, 1960).

Distinto es cuando se trata de encuestas de boca de urna y sus resultados se difunden antes de que termine la elección: en este caso, quien todavía no votó tiene más información que el que ya lo hizo, y esto puede implicar cambios en la decisión del voto. 
Otra interesante y bien diferente mirada sobre la relación entre opinión pública y medios de comunicación es la de Noelle-Neumann (1995), con su muy conocida teoría de la espiral del silencio. Esta politóloga alemana, fundadora del Institut Demoskopie Allensbach, desarrolla sus hipótesis a partir del fracaso de las encuestas en las elecciones federales de 1965 y 1972 en Alemania, donde muy pocos días antes, y sin que las encuestas lo anticiparan, se produce un cambio importante en la dirección del voto. Según la autora, entonces, la opinión pública es el resultado de la interacción entre los individuos y su entorno. Desde una perspectiva psicosociológica -el temor al aislamiento- define a la opinión pública como aquellas opiniones que pueden ser expresadas públicamente sin riesgo de sanciones. Así, la disposición de un individuo a exponer en público su punto de vista varía según la apreciación que hace acerca del reparto de las opiniones en su entorno social: un clima hostil tiene un efecto negativo sobre la voluntad de expresar los propios puntos de vista. La difusión de las encuestas contribuye a generar un clima de opinión. Desde esta perspectiva, los medios de comunicación masiva -y la publicación de las encuestas- crean opinión pública en tanto constituyen el entorno cuya presión desencadena la confrontación, la sumisión o el silencio.

En cualquier caso, es evidente que las encuestas tienen un carácter performativo: no sólo describen su objeto de estudio, también contribuyen a crearlo y modificarlo; no sólo miden la opinión pública, también participan en su formación y cambio. Si nos remitimos estrictamente a las encuestas preelectorales y a su uso, que podemos llamar privado -esto es, como insumo de una campaña-, es evidente que, como cualquier elemento de una campaña política, se hacen para influir sobre la decisión del votante. $\mathrm{Si}$, en cambio, nos remitimos a su uso público -esto es, al 
que deriva de su difusión-, puede pensarse que, cuando sus resultados reingresan a la sociedad y son tenidos en cuenta por los diferentes actores, "ayudan a cambiar las características del fenómeno observado o a originar fenómenos nuevos" (Cordero, 2009). Pero esto no las hace sospechosas; simplemente, nos habla de la complejidad del campo.

Un segundo campo de ambigüedades en que se mueven las encuestas tiene que ver con su capacidad de predecir, producto del uso de las técnicas probabilísticas, y la alta probabilidad de cometer errores. Y no se trata sólo del error "conocido", el normalmente denominado "margen de error" y que tiene que ver con el tamaño de la muestra y su nivel de confianza. Existen también errores menos controlables, como aquellos que derivan de problemas de cobertura -segmentos de la población que no tienen la posibilidad de ser incluidos en la muestra-, sea por el costo que a veces tiene llegar a ciertos segmentos (población rural, por ejemplo), sea por la carencia, desactualización o poca confiabilidad de los datos oficiales. También están aquellos errores que resultan de las limitaciones de una metodología basada en un cuestionario: sabemos que distintas fraseologías o escalas pueden dar resultados diferentes, o que el lugar en el cuestionario de una determinada pregunta puede mostrar distribuciones de frecuencias bien disímiles. Finalmente, están aquellos errores que tienen que ver con la naturaleza intrínseca de las encuestas: la existencia -en todas las sociedades y en todo tipo de régimen- de personas que se niegan a dar su opinión a través de una encuesta, o de la propensión de los individuos a dar respuestas "políticamente correctas" o socialmente esperadas.

En tercer lugar, las encuestas se mueven también entre la rapidez exigida por la industria de la producción de información o por la actividad de la consultoría política, y 
la complejidad que exige el análisis de su objeto, el comportamiento humano. Como dice un conocido consultor político: "Los consultores vivimos la vorágine de las campañas y del poder. Trabajamos para cambiar la realidad $\mathrm{y}$, para lograrlo, necesitamos hacer estudios a un ritmo vertiginoso... Algunos querríamos escribir En busca del tiempo perdido, pero necesitaríamos muchos meses para recorrer los caminos de Swann y no los tenemos" (Durán Barba, 2009). Se trata entonces de una actividad que muchas veces reclama instantaneidad en la producción de datos -y esta instantaneidad puede entrar en colisión con los estándares metodológicos-, pero que a la vez está poblada de hipótesis múltiples y encontradas, de factores intervinientes muy difíciles de investigar, de las incertidumbres propias de acercarse a un fenómeno tan intrincado como los procesos socioculturales.

Las encuestas se mueven, por último, entre la necesidad de interactuar con el campo académico (todo encuestador debe actualizar y renovar sus conocimientos teóricos y sus competencias y destrezas técnicas), y el hecho de que la infraestructura que exigen sus estudios es muy compleja y costosa. La investigación por encuesta exige, además de un conjunto de competencias y destrezas, una serie de condiciones de trabajo (dedicación full time a la profesión, trabajo en equipo) no siempre fáciles de lograr en las carreras de estudios sociales. Esto le ha dado, como veremos más adelante, un papel bastante marginal a la academia en la producción de información empírica, y han sido más bien los partidos políticos y las empresas los encargados de financiar este tipo de trabajos.

Nos encontramos, entonces, con una actividad que resulta públicamente sobreexpuesta -y criticada a la vez que requerida-, a la que se le reclama capacidad predictiva (siendo que las ciencias sociales hace ya mucho tiempo han dejado de postular cualquier pretensión de exactas), 
y a la que se le exige urgencia, cuando lo que se precisa es paciencia y minuciosidad en el tratamiento y el análisis de la información.

\section{Una industria "en ebullición"}

Por otro lado, estamos frente a una actividad que ha crecido notablemente a lo largo de estas últimas décadas. En efecto, cada vez son más las encuestas que se realizan y publican, y cada vez también son más los actores que producen, demandan y consumen estudios de opinión pública. Las encuestas no sólo las usan los políticos en campaña; también las instituciones encargadas de ejecutar políticas públicas recurren a ellas para implementar o evaluar planes o proyectos. La sociedad -o al menos algunos segmentos de ella- cada vez está más interesada en este tipo de información y, consecuentemente, los medios en publicarla; las instituciones con peso social -empresas, organismos multilaterales, etc.- procuran informarse sobre las tendencias políticas y culturales.

Aunque por desgracia son muy pocos los datos que existen, una serie de evidencias permite afirmar que estamos frente a una industria que viene experimentado, en la Argentina y en América Latina, un crecimiento notable. Entre otros indicadores, puede mencionarse, además del aumento de la producción y publicación de encuestas, el creciente interés por la capacitación -cursos de especialización y de posgrado-, el desarrollo de estudios regionales (Latinobarómetro, Iberobarómetro, Barómetro Latinoamericano de Gobernabilidad, etc.), la inclusión de los países latinoamericanos en los grandes estudios de opinión pública internacional (Estudio Mundial de Valores, World Poll, los estudios del Pew Center, Globe Scan, etc.) y finalmente el surgimiento, aunque precario, de ciertas 
instituciones que aspiran a nuclear a los especialistas en el tema. ${ }^{5}$

Al mismo tiempo, se trata de una actividad relativamente nueva en el continente: las dictaduras de los años 1970 interrumpieron de manera brusca el proceso de acumulación en ciencias sociales que, con mayor o menor grado, venía dándose en la mayoría de los países latinoamericanos. ${ }^{6}$ En la mayoría de los países de América Latina, la investigación social empírica se va a recluir en algunos pocos ámbitos alternativos, ${ }^{7} \mathrm{y}$ en ciertos casos, en las empresas de investigación de mercado. Puede decirse que durante esos años, la investigación empírica, en la mayoría de los países de América Latina, fue escasa, asistemática y poco ligada a la academia.

Será recién con la vuelta de la democracia que las encuestas comienzan a instalarse como un campo específico dentro de las ciencias sociales. Hoy estamos, efectivamente, frente a una actividad que se identifica con un nombre propio -el de opinión pública (Mora y Araujo, 2009)- y que,

5 Un ejemplo de estos desarrollos es la creciente actividad, en la región, de WAPOR (World Association for Public Opinion Research), una asociación cuyo objetivo es promover y asistir el desarrollo y la publicación de la investigación en opinión pública. El Primer Congreso Latinoamericano de Opinión Pública, en abril de 2007, que se llevó a cabo en la ciudad de Colonia del Sacramento, Uruguay, fue seguido de una serie de congresos (Lima, Perú, en octubre de 2008; Querétaro, México, en abril de 2010; Belo Horizonte, Brasil, en mayo de 2011) que reunieron por primera vez, a nivel latinoamericano, a una comunidad de investigadores de opinión pública. Estimulados por el éxito de estos eventos, un grupo de profesionales acaba de crear, en Buenos Aires, la primera sede de WAPOR Latinoamérica, entidad que se propone desarrollar a nivel local y regional los objetivos de WAPOR (ver wwww.waporlatinoamerica.org). Experiencias como las de Gino Germani desde el Instituto de Sociología en la Argentina, o de Eduardo Hamuy en Chile desde el departamento de Sociología de la Universidad de Chile son sólo algunos ejemplos de experiencias valiosas que fueron discontinuadas como consecuencia de los golpes militares

7 En la Argentina, por ejemplo, en la Fundación Bariloche. 
como tal, ha creado sus propios criterios de funcionamiento: métodos científicos de recolección de datos, tecnologías de procesamiento de información, nuevos lenguajes y narrativas para designar y explicar lo social, y desarrollo de instituciones y especialistas orientados a producir este conocimiento (Cordero, 2009).

Siempre en relación con el desarrollo de los estudios de opinión pública en las últimas décadas en América Latina, cabe tener en cuenta los datos de una encuesta realizada por WAPOR Latinoamérica entre profesionales y académicos latinoamericanos ligados al estudio de la opinión pública. ${ }^{8}$ Diseñada con el objetivo de hacer un relevamiento básico de la situación de la profesión y de las críticas que recibe, la encuesta muestra, en primer lugar, que los estudios de opinión pública política -básicamente encuestas electorales-, así como la realización de encuestas boca de urna son, desde la década de 1980, un tipo de información que la gente está habituada a recibir a lo largo de una competencia electoral.

Agradecemos especialmente la participación de Luis Eduardo González en la confeccion del cuestionario y de MBC MORI en la realización del trabajo de campo. Para el diseño de la muestra se partió de la lista de miembros latinoamericanos de WAPOR, y se utilizó la técnica de "bola de nieve", pidiéndole a referentes clave de la actividad que sugirieran nombres de colegas. De esa forma se confeccionó una base de datos que incluyó 200 nombres. Respondieron al cuestionario 139 personas de los principales países de América Latina (Argentina, Brasil, Chile, Colombia, Costa Rica, Ecuador, Honduras, México, Paraguay, Perú, República Dominicama, Uruguay y Venezuela). La encuesta se basó en un cuestionario de 15 preguntas cerradas y se aplicó vía Internet. El trabajo de campo se hizo entre los días 29 de marzo y 30 de mayo de 2010 . 


\section{Gráfico 1: Tiempo que hace que se publican encuestas y que se realizan boca de urna}

Preg: “¿Desde cuándo se difunden con cierta regularidad encuestas de opinión pública política de su país en medios de comunicación masiva?"

Preg.s: "¿Desde cuándo se realizan encuestas de boca de urna y/o de anticipo de escrutinio en su país en elec

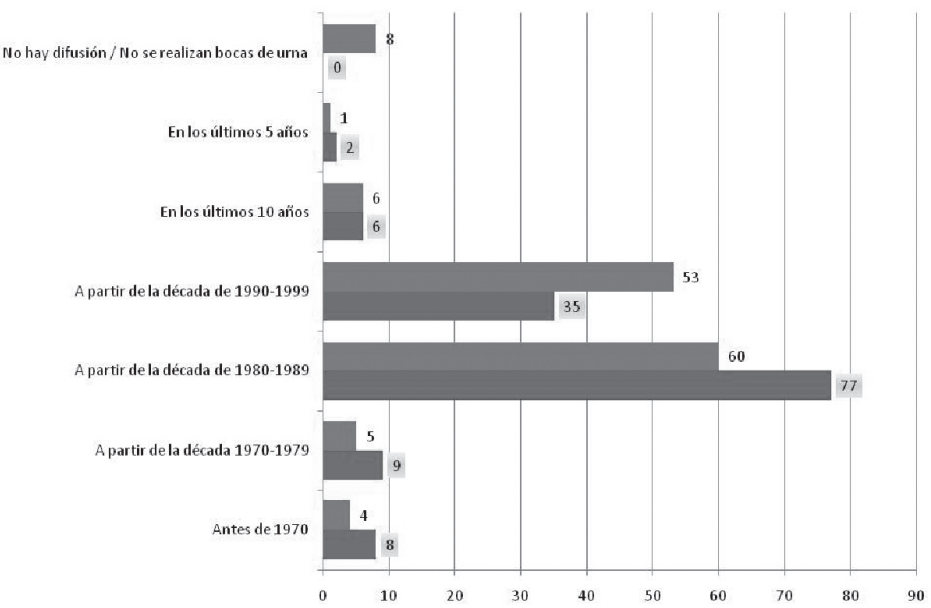

Por otro lado, y siempre desde la perspectiva de los encuestadores, estamos efectivamente frente a una actividad que responde a los estándares internacionales y a cuyo cargo están especialistas, personas con formación. 


\section{Gráfico 2: Evaluación de la calidad de las encuestas de OP}

Preg.: "En su opinión personal, las mejores encuestas de opinión pública política en su país..."

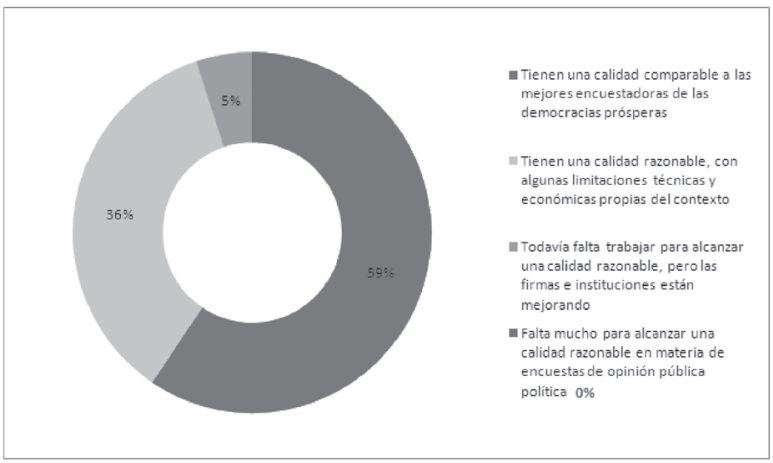

Base: total entrevisados $(n=139)$

Fuente: WAPOR Latinoamérica

\section{Gráfico 3: Perfil profesional de los encuestadores conocidos}

Preg.: “¿Quiénes son las principales "caras visibles" de esas firmas?"

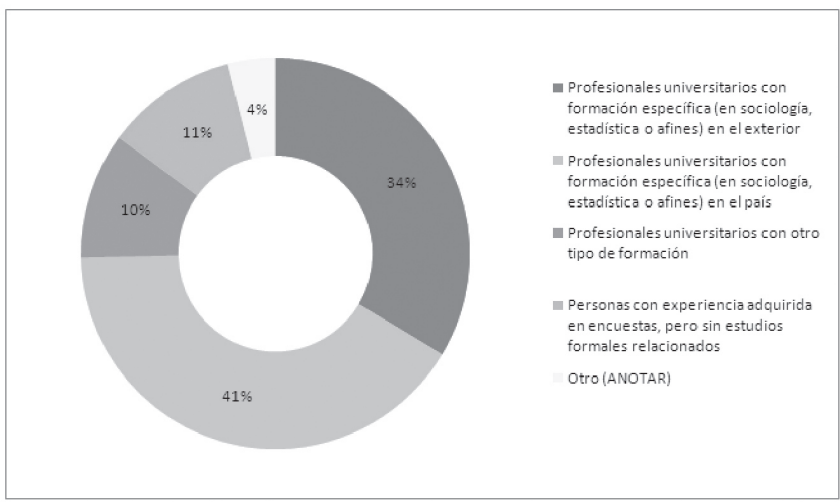


Sin embargo, las encuestas son fuertemente criticadas (el 73\% de los encuestados afirma que las críticas son muy frecuentes), y estas críticas provienen de distintos sectores: sobre todo de los medios de comunicación, pero también de los partidos políticos, los gobiernos y, en menor medida, la academia. La principal crítica que según los encuestadores reciben las encuestas es la de falsear o esconder resultados por razones partidistas o económicas.

Gráfico 5: Principales críticos de las encuestas

Preg.: "¿Quiénes son los críticos más frecuentes de las encuestas?” (respuesta mútiple)

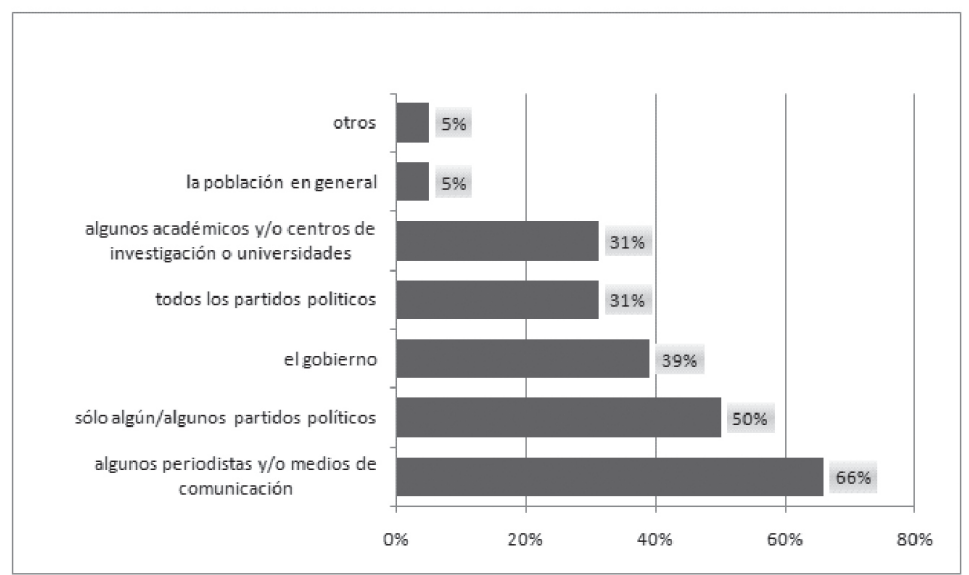




\section{Gráfico 6: Tipo de críticas a las encuestas y a los encuestadores}

Preg : “¿Cuál es la naturaleza más frecuente de las críticas a las encuestas y las firmas encuestadoras?”

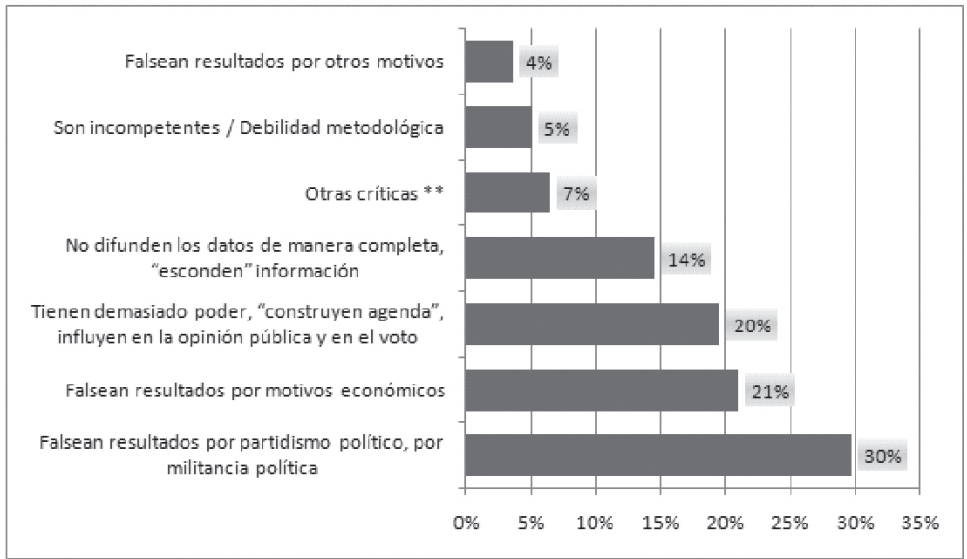

** En "Otras críticas" se observa en muchos casos respuestas que combinan el falseamiento de resultados por motivos económicos y políticos a la vez.

Ahora bien, cabe destacar que las suspicacias y las críticas no provienen sólo de los públicos externos. Así, la mayoría de los entrevistados afirma que existen polémicas entre las firmas que difunden datos de opinión pública política, y que las críticas que se formulan se centran, otra vez, en falsear o esconder información, en general por razones que tienen que ver con la militancia política. 


\section{Gráfico 7: Relaciones entre las firmas encuestadoras}

Preg.: "Pensando en todas las firmas que difunden resultados de encuestas de opinión pública política, ¿hay polémicas o críticas entre las distintas firmas?"

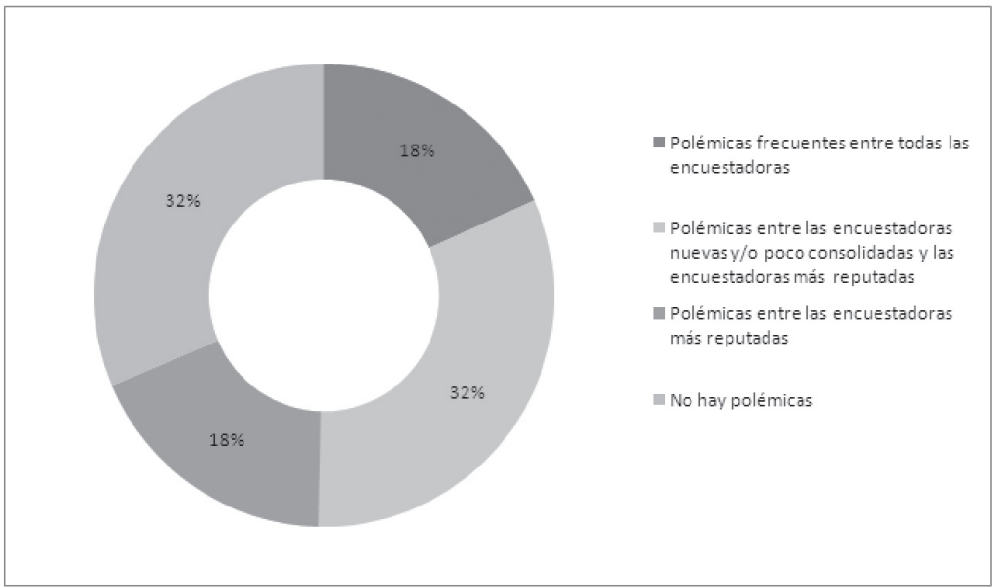




\section{Gráfico 8: Tipos de críticas entre las firmas encuestadoras}

Preg.: “¿Cuál es la naturaleza más frecuente de las críticas entre firmas encuestadoras?"

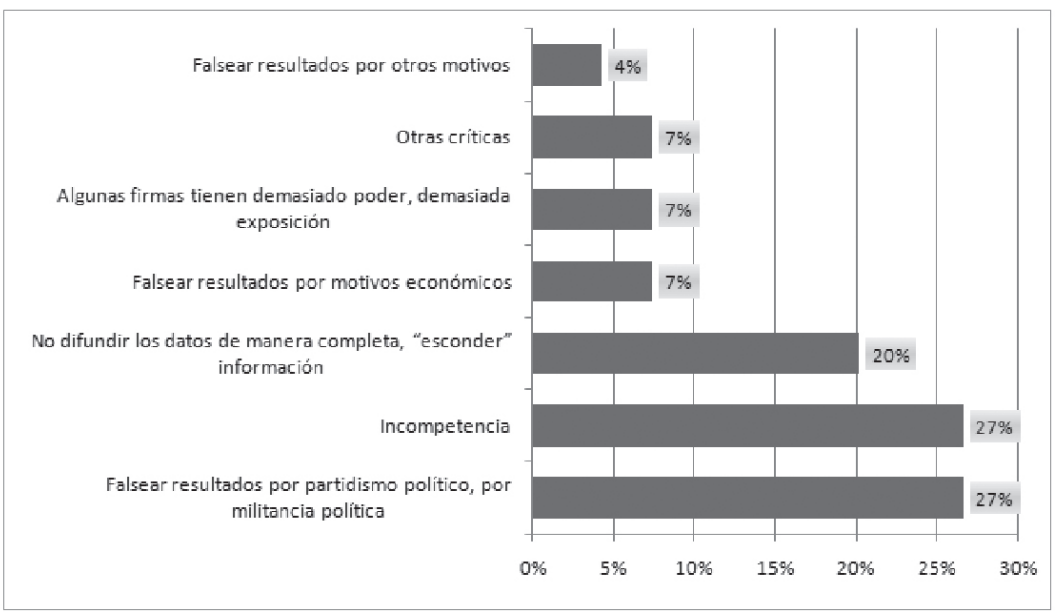

Nos encontramos entonces frente a una industria en franco crecimiento pero, según sus propios protagonistas, con reglas poco claras. Una actividad muy ligada a la lógica de la estrategia electoral y de la producción de acontecimientos, donde la presencia de la academia -como resultado, más que nada, de cambios institucionales bruscos y frecuentes- ha sido baja. En síntesis, una disciplina débilmente institucionalizada, que se ha ido incorporando a las ciencias sociales de manera bastante "salvaje" (Brunner, 1985), que no ha cerrado aún su ciclo de profesionalización y que, en consecuencia, todavía debe dar pasos para construir su legitimidad. 


\section{Una profesión débilmente institucionalizada}

Es verdad que el consenso acerca de la contribución de las encuestas a las democracias modernas es bajo. Según algunos (Huneeus, 2007), al dar el mismo peso a cada una de las voces que conforman la ciudadanía, permiten corregir la desigualdad política que existe en las democracias. Para otros -Bourdieu, por ejemplo- las encuestas son un mero instrumento de los sectores dominantes para "construir" aquello que no existe: la opinión pública (Bourdieu, 1990).

En cualquier caso, es bastante evidente que las encuestas intervienen en la lógica de funcionamiento de la democracia. Puede decirse, por ejemplo, que usarlas para elegir candidatos debilita la legitimidad de los partidos, o que la preocupación permanente por los niveles de aprobación pone bajo sospecha la legitimidad presidencial durante el período de gobierno (Navia, 2009, p. 115).

La caída de la importancia de los partidos políticos como principal canal de expresión de las demandas sociales ha impactado, sin dudas, sobre el papel de las encuestas. Series longitudinales y datos comparados muestran que cada vez es menor la participación de las personas en las organizaciones partidarias, ${ }^{9}$ que vienen disminuyendo muy significativamente los índices de identificación partidaria ${ }^{10}$ y que es muy baja la confianza de la población en los partidos políticos. ${ }^{11}$ Esto hace que prever el comportamiento político sea hoy mucho más difícil que hace unas décadas:

Ver datos Latinobarómetro en www.latinobarómetro.org.

A mediados de los años 1980, estos índices de identificación partidaria -afiliación más simpatía-incluían, en la Argentina, a aproximadamente un $40 \%$ de la población mayor de 18 años. Hoy estos niveles no alcanzan el $20 \%$.

11 Los datos comparativos del Worl Values Survey muestran, a nivel latinoamericano, que el Uruguay es el país donde es más alto el nivel de confianza en los partidos (36\%), Chile tiene niveles intermedios (28\%) y Argentina presenta los niveles más bajos (7\%). (Ver Segovia, 2009.) 
de hecho, cada vez son más los ciudadanos que, por ejemplo, deciden su voto a partir de las campañas políticas, o que votan candidatos que vienen de fuera del campo de la política, o que, de elección en elección, fluctúan de un partido a otro.

Cuando es cada vez más difícil tener certezas, cuando resulta cada menos previsible el comportamiento político de los ciudadanos, se les pide a las encuestas que den certezas y se las cuestiona cuando se equivocan. Ahora bien, ¿es tan evidente que la legitimidad de las encuestas debería estar dada por su carácter predictivo? ¿Es eso acaso lo que las hace más fiables? No acertar el resultado -muchas veces previsto y publicado varios días antes de las elecciones-, ¿descalifica las encuestas, aun cuando éstas hayan sido hechas siguiendo los requerimientos técnicos?

La legitimidad de las encuestas es, volviendo a Wolton, una legitimidad técnica. Pero esta legitimidad técnica, como la de cualquier saber, es una construcción, y como tal incluye o debe incluir la construcción de consensos sobre la necesidad y la justeza de ese discurso y de las técnicas a él asociadas (Vommaro, 2008, p. 16). Todo proceso de “justificación" de una disciplina científica en el campo intelectual pone en juego, por lo mismo, estrategias por parte de los actores involucrados: generar un saber relativamente diferenciado y con mecanismos de validación propios y socialmente reconocidos como legítimos implica la necesidad de que la comunidad científica, que es ante todo una red de comunicación, sea la encargada de controlar la producción, de fijar los estándares de evaluación del trabajo disciplinario, de establecer la jerarquía de méritos y de asegurar la vigencia del particular ethos al cual debe adscribirse el comportamiento de los científicos (Neiburg y Plotkin, 2004; Brunner, 1985).

El desarrollo de la disciplina de los sondeos no estuvo presidido por un programa; no fue, en modo alguno, el 
resultado de un proceso de planificación, sino un producto gradual, contradictorio y pleno de rupturas. La democracia le dio un impulso notable a la actividad y un lugar destacado. Pero los procesos de generación de reglas, los mecanismos de comunicación y de control en el campo de la actividad no existieron o fueron muy limitados. Hoy, como dice Mora y Araujo, es absolutamente imprescindible un desarrollo profesional que apunte a "mejorar estándares de calidad, discutir abiertamente los problemas metodológicos, intercambiar más de lo que está ocurriendo entre la profesión y la academia, y profundizar los intentos de comprensión del papel de la profesión en la vida social y política de nuestro tiempo" (Mora y Araujo, 2009, p. 66). Esto, y generar mecanismos que ayuden a la transparencia, contribuirían sin dudas a legitimar el campo de los estudios de opinión.

En términos más específicos, es necesario definir y acordar una agenda de problemas y soluciones que parta de una actitud comprensiva de los problemas de la actividad -alguno de los cuales he tratado de plantear en estas páginas-, más que de una posición normativa. Como propuesta inicial y teniendo en cuenta "reclamos" ya planteados por diversos referentes de la actividad, podría trabajarse en las siguientes direcciones:

1. Definir y llevar adelante programas de "adiestramiento formal" sobre bases institucionales adecuadas (cátedras, carreras, facultades).

2. Avanzar en la dirección de construir un banco de datos, de carácter local o regional, que ponga la información de las encuestas -una información muy valiosa pero costosa- a disposición de la comunidad científica. Los datos son recursos no agotables: pueden revisarse una y otra vez; nuevos investigadores les encontrarán más riqueza, nuevas dimensiones, más ideas y mensajes. "Reutilizar y 
hacer públicos los datos es no sólo legítimo, sino inclusive necesario" (www.waporlatinoamerica.org). ${ }^{12}$

3. Encarar el proceso de relacionamiento entre la investigación de opinión pública y los medios de comunicación, asegurando el tratamiento más profesional de la información y el mejoramiento de la calidad en el diseño, uso y difusión de encuestas en los medios (más allá de las decisiones editoriales de los medios -algo sobre lo cual es difícil intervenir-, la deficiente difusión de los resultados depende muchas veces de la falta de preparación de los periodistas, o de la escasa información técnica que entregan los encuestadores. Se podría pensar en avanzar en la conformación de ciertos protocolos, de modo de lograr que los procedimientos de la investigación sean más transparentes, más visibles y más públicos y controlables).

4. Conocer mejor los procesos internos del campo, su historia y sus componentes, dando cuenta de sus prácticas de producción: tipo de instituciones que las realizan, características técnicas de las encuestas realizadas, tipo de información publicada, relaciones y articulaciones con otros actores involucrados. ${ }^{13}$ Reconstruir la histo-

En los países desarrollados, los archivos nacionales de opinión pública existen desde hace décadas, crecen incesantemente mediante aportes de instituciones de investigación públicas y privadas, son ampliamente accesibles por parte de la comunidad académica e interesados no especialistas, y gozan de un prestigio indiscutido. En nuestros países, la idea de que los frutos de la investigación deben abrirse a la discusión y compartirse con la comunidad es aún novedosa, aunque por fortuna gana creciente aceptación, e incluso comienza a reclamarse como una necesidad (www.waporlatinoamerica.org).

13 El trabajo de Vommaro sobre las encuestas en la Argentina entre 1983 y 1999 es una excelente descripción de cómo se fue constituyendo ese campo en nuestro país (Vommaro, 2008). Ahora bien, este trabajo no cuenta con información global sobre la industria ni con datos de carácter cuantitativo. En ese sentido, las investigaciones realizadas por Rodrigo Cordero y Gonzalo Tapia desde la Universidad Diego Portales son un 
ria de la industria de la opinión pública en América Latina permite ampliar el campo de conocimiento respecto del impacto de los estudios de opinión en la vida pública y política de la región (Aguiar, 2009).

5. Generar espacios para la validación de ideas y de prácticas: esto significa, básicamente, establecer ámbitos de encuentro y de reflexión (congresos, seminarios, jornadas, work shops, etc.), y promover publicaciones especializadas con estándares de calidad a nivel internacional.

6. Avanzar en la formación de una comunidad regional: América Latina tiene problemas que le son comunes. Crear un círculo latinoamericano estructurado por instituciones -publicaciones, encuentros, congresos, etc.- resulta fundamental para poder reflexionar sobre estos problemas, para avanzar en el conocimiento de nuestras sociedades y para conocer mejor las problemáticas específicas ligadas a nuestra profesión. Estas redes deberían incluir también a los investigadores de mercado.

7. Avanzar en la formación de una instancia institucional que acuerde normas técnicas y éticas que comprometan a todos los asociados y que aporten a la transparencia de la información. Transparentar la información significa entregarla en formatos que permitan hacer una evaluación de qué se está produciendo, cómo y para quién. También implica incorporar a este proceso a otros actores, entre ellos los medios de comunicación.

\section{Para terminar}

Con distintas modalidades, hoy las encuestas de opinión pública y los estudios preelectorales están instalados

modelo más que apropiado para pensar un programa de investigación que reconstruya la historia de las encuestas de opinión. 
en todas las democracias consolidadas o recientes. La discusión sobre ellas se centra, en todo caso, no en su validez, en su capacidad para describir, explicar e incluso predecir ciertos comportamientos, sino básicamente en los requerimientos que su uso implica, en las reglas que guían su funcionamiento, en la relación entre el mundo académico y la actividad comercial, en la vinculación de las encuestas con los medios de comunicación y en su capacidad para impactar sobre las decisiones políticas.

Conformar una comunidad profesional y académica capaz de controlar la excelencia en la producción de encuestas, y desarrollar espacios para la reflexión serena y ajustada acerca de la naturaleza de esta piel social que recubre e influye en las opiniones y creencias de cada uno, es, sin duda, además de un tema de la agenda de nuestros tiempos, una forma de no abandonar al lugar común uno de los mecanismos más apasionantes que la ciencia social moderna nos ha legado.

\section{Bibliografía}

Aguiar, Cesar (2009), "Apuntes para una historia de los estudios de opinión pública en América Latina”, en Rodrigo Cordero (ed.), La sociedad de la opinión. Reflexiones sobre las encuestas y cambio político en democracias, Chile, Ediciones Universidad Diego Portales.

Alonso, B.; Cabrera, D.; y Tesio, M. A. (2009), "Sondeos, votos y voces, aportes para un debate político y técnico", en María Braun y Cecilia Straw (eds.), Opinión pública: una mirada desde América Latina, Buenos Aires, Emecé.

Bourdieu, Pierre (1990), "La opinión pública no existe", Sociología y cultura, México, Grijalbo. 
Braun, María; Catterberg, Gabriela; y Rovner, Helena (2009), "Reflexiones sobre el campo de la opinión pública", Revista Espacios Politicos, Buenos Aires.

Braun, María y Rovner, Helena (1999), "Encuestas de opinión: entre el lugar común y la construcción del saber", Lo que vendrá, año 4, núm. 8, Buenos Aires, Facultad de Ciencias Sociales de la Universidad de Buenos Aires.

Brunner, José Joaquín (1985), "Los orígenes de la sociología profesional en Chile", Documento de Trabajo, Programa FLACSO / Santiago de Chile, núm. 260, septiembre de 1985.

Cordero, Rodrigo (2009), "Dígalo con números: la industria de la opinión publica en Chile", en Rodrigo Cordero (ed.), La Sociedad de la opinión, Reflexiones sobre las encuestas y cambio político en democracias, Chile, Ediciones Universidad Diego Portales.

Cordero, Rodrigo y Tapia Horment, Gonzalo (2009), "Entre la ciencia, la política y el mercado: la construcción de la industria de la opinión pública en Chile", en María Braun y Cecilia Straw (eds.), Opinión pública: una mirada desde América Latina, Buenos Aires, Emecé. Donsbach, Wolfgang (2001), Who's Afraid of Election Polls? Normative and Empirical Arguments for the Freedom of Pre-Election Surveys, Ámsterdam, ESOMAR.

Durán Barba, Jaime (2009), "Los encuestadores, los consultores y sus mitos", en María Braun y Cecilia Straw (eds.), Opinión pública: una mirada desde América Latina, Buenos Aires, Emecé.

ESOMAR/WAPOR(2003), Guide to Opinion Polls, Foundation for Information. Disponibleenlínea:www.esomar.org/.../ ESOMAR_Codes\&Guidelines_OpinionPolling_v5 Huneeus, Carlos (2007), "Democracia versus encuestocracia”, en Revista Mensaje, Santiago, Chile.

Lazarsfeld, P. F. y Katz, E. (1960), La influencia personal, Santiago, Andrés Bello. 
Mora y Araujo, Manuel (2009), "La opinión pública en la Argentina: de la sociología académica a la profesionalización", en Rodrigo Cordero (ed.), La sociedad de la opinión. Reflexiones sobre las encuestas y cambio politico en democracias, Chile, Ediciones Universidad Diego Portales.

Navia, Patricio (2009), “Encuestas, desempeño electoral y preferencias electorales, 1990-2007", en Rodrigo Cordero (ed.), La sociedad de la opinión. Reflexiones sobre las encuestas y cambio político en democracias, Chile, Ediciones Universidad Diego Portales.

Neiburg, F. y Plotkin, M. (2004), "Intelectuales y expertos, hacia una sociología histórica de la producción del conocimiento sobre la sociedad en la Argentina", en Neiburg y Plotkin (comp.), Intelectuales y expertos. La constitución del conocimiento social en la Argentina, Paidós, Buenos Aires.

Neuman, Noelle (1995), "La espiral del silencio. Una teoría de la opinión pública”, en Ferry, Wolton et al., El nuevo espacio público, Barcelona, Gedisa Editorial.

Segovia, Carolina (2009), “¿Crisis de la política en Chile? Percepciones y valoraciones de los partidos", en Rodrigo Cordero (ed.), La sociedad de la opinión. Reflexiones sobre las encuestas y cambio político en democracias, Chile, Ediciones Universidad Diego Portales.

Vommaro, Gabriel (2008), “Lo que quiere la gente”, Los sondeos de opinión y el espacio de la comunicación política en Argentina (1983-1999), Buenos Aires, Prometeo.

Wolton, Dominique (1995), "La comunicación política: construcción de un modelo", en Ferry, Wolton et al., El nuevo espacio público, Barcelona, Gedisa Editorial. 